\title{
From deterministic to stochastic surrender risk models: impact of correlation crises on economic capital
}

July 7,2010

\author{
Stéphane Loisel ${ }^{*}$ \\ Université de Lyon, Université Claude Bernard Lyon 1, Institut de Science \\ Financière et d'Assurances, 50 Avenue Tony Garnier, F-69007 Lyon, France
}

\section{Xavier Milhaud}

Université de Lyon, Université Claude Bernard Lyon 1, Institut de Science

Financière et d'Assurances, 50 Avenue Tony Garnier, F-69007 Lyon, France

Axa Global Life, 40 rue du Colisée, 75008 Paris

\begin{abstract}
In this paper we raise the matter of considering a stochastic modeling of the surrender rate instead of the classical S-shaped deterministic curve (in function of the spread), still used in almost all insurance companies. A stochastic model in which surrenders are conditionally independent with respect to a S-curve disturbance would be tempting in some extreme scenarii, especially to address the question of the lack of data. However, we explain why this conditional independence between policyholders, which has the advantage to be the simplest assumption, looks particularly maladaptive when the spread increases. Indeed the correlation between policyholders' decisions is most likely to increase in this situation. We suggest and develop a simple model which integrates those phenomena. With stochastic orders it is possible to compare it to the conditional independence approach qualitatively. In an partially internal Solvency II model, we quantify the impact of the correlation phenomenon on a real life portfolio for a global risk management strategy.
\end{abstract}

Surrender risk, also called lapse risk represents one of the main dangers faced by a life insurance institution. It corresponds to the risk that many policyholders surrender their contract earlier than expected and choose to reinvest

* Corresponding author

Email addresses: stephane.loisel@univ-lyon1.fr (Stéphane Loisel), xavier.milhaud@gmail.com (Xavier Milhaud). 
their money in another product or in some project. Because fees are charged throughout the duration of the contract, the insurer may not have enough time to charge the fees in the case of early surrenders. Massive early surrenders might also cause important liquidity issues and of course a loss of market share. Many policyholders surrender their life insurance contract every year, mainly to finance a project (building a new house, purchasing a new car, ...) or because the tax incentive delay ( 8 years in France) has been reached. Insurers are used to forecasting lapse rates, which may be explained by different factors (see Milhaud, Loisel \& Maume-Deschamps (2010) for more details). In Solvency II, internal or partially internal models are being developed by many companies. They have to go from a deterministic model, often based on a so-called S-shaped lapse rate curve to a stochastic model. The S-shaped curve corresponds to the lapse rate expressed as a function of the difference $\Delta r$ between the interest rate given by the contract and the one that the policyholder could obtain somewhere else in the market. The idea that practitioners have followed is that even if $\Delta r$ is very small, some policyholders are going to surrender their contract for tax reasons or to fund a personal project, that the lapse rate is increasing in $\Delta r$, and that even if $\Delta r$ is very large, some policyholders are going to stay in the portfolio because they do not really pay attention to the market evolution. The problem with this S-shaped curve is that one has not observed policyholders' behavior in the extreme situation in which $\Delta r$ is very large.

To build a stochastic model, given this lack of information, one must more rely on thought experiments than on statistical data (which simply does not exist). It may be tempting for internal model designers to use a Gaussian distribution around the value of the lapse rate in the S-shaped, deterministic curve to describe stochastic surrender risk. In this paper, we explain why it may be preferable to use a bi-modal distribution, due to the likely change in the correlation between policyholders' decisions in extreme scenarios. This change of correlation in extreme situation, called correlation crisis in Biard et al. (2008), Loisel et al. (2010) and Loisel (2008) prevents us from applying the classical Gaussian approximation based on the central limit theorem. This theorem holds when decisions of policyholders are independent. Here, this would be the case only given a certain factor that would incorporate the level of information of policyholders and the reputation of the company and of the insurance sector. This factor should be a key element in the internal model to understand the correlation of surrender risks with other risks like market risk or default risk.

Correlation crises have followed the sub-prime crisis in both stock markets and credit derivatives market: in many cases, correlation increases as things go very bad. For surrender risk, it is likely that an extreme situation in interest rates markets would lead either to massive surrenders, or to almost normal lapse rates, depending on political declarations and on other factors: for example, one of the first things that leaders of developed countries said at the beginning of the last crisis was: We guarantee bank deposits and classical 
savings products. This leads to anticipate policyholders' behavior more like a $0-1$ law than according to a bell-shaped unimodal distribution. In this paper, we propose a basic model that takes into account correlation crises: as $\Delta r$ increases, correlation between policyholders' decisions increases, and one goes (continuously) from a bell-shaped distribution in the classical regime to a bi-modal situation when $\Delta r$ is large.

\section{The model}

Assume that when $\Delta r$ is zero, policyholders behave independently and that the average lapse rate is $\mu(0)$, and that when $\Delta r$ is very large $(15 \%$, say), the average lapse rate is $1-\epsilon$ with $\epsilon$ very small, and that the correlation between individual decisions is $1-\eta$, with $\eta$ very small. A model that captures this simple features is the following: let $I_{k}$ be the random variable that takes value 1 if the $k^{\text {th }}$ policyholder surrenders her contract. Assume that

$$
I_{k}=J_{k} I_{0}+\left(1-J_{k}\right) I_{k}^{\perp}
$$

where $J_{k}$ corresponds to the indicator that the $k^{\text {th }}$ policyholder follows the market consensus (copycat behavior). The random variable $J_{k}$ follows a Bernoulli distribution whose parameter $p_{0}$ is increasing in $\Delta r$, and $I_{0}, I_{1}^{\perp}, I_{2}^{\perp}, \ldots$ are independent, identically distributed random variables, whose parameter $p$ is also increasing in $\Delta r$. This means that the surrender probability increases with $\Delta r$, and that the correlation (Kendall's $\tau$ or Spearman $\rho$ ) between $I_{k}$ and $I_{l}$ (for $k \neq l)$ is equal to $P\left(J_{k}=1 \mid \Delta r=x\right)$ when $\Delta r=x$, and that in general (without conditioning) the correlation between $I_{k}$ and $I_{l}$ (for $k \neq l$ ) is equal to

$$
\int_{0}^{+\infty} P\left(J_{k}=1 \mid \Delta r=x\right) d F_{\Delta r}(x) .
$$

This is because given that $\Delta r=x, I_{k}$ and $I_{l}$ (for $k \neq l$ ) admit a Mardia copula (linear sum of the independent copula and of Fréchet upper bound) ${ }^{1}$. For a portfolio of 20000 policyholders, the Gaussian assumption is not too bad for the case where $\Delta r=0$. We show here with realistic values of the S-shaped curve how this bell-shaped curve progressively evolves as $\Delta r$ increases and at some point $\Delta r=x_{0}$ becomes bi-modal. McNeil et al. (2005) perfectly illustrates the problem of correlation risk and its consequences on tail distribution in a general context.

\footnotetext{
$\overline{1}$ Here the copula of $I_{k}$ and $I_{l}$ (for $k \neq l$ ) is not unique as their distributions are not continuous.
} 


\section{Interpretation of the model}

The S-shaped curve of the surrender rate in function of $\Delta r$ on Figure 1 shows that the less attractive the contract is, the more the policyholder tends to surrender it. Obviously the surrender rate average is quite low in a classical economical regime (Region 1, low $\Delta r$ on Figure 1), but is significantly increasing as $\Delta r$ increases. Indeed when interest rates rise, equilibrium premiums decrease and a newly acquired contract probably provides the same coverage at a lower price: the investor acts as the opportunity to exploit higher yields available on the market. On the contrary, if the interest rates drop then the guaranteed credited rate of the contract may be (when it is possible) lowered by the insurer (for financial reasons or to stimulate the policyholder to surrender).

By consequence, Region 1 in Figure 1 illustrates the case corresponding to independent decisions of policyholders (here the correlation tends to 0) whereas Region 2 corresponds to much more correlated behaviors (correlation tends to 1 in this situation) because of a crisis for instance. The underlying idea of the paper is that as long as the economy remains in "good health", the correlation between policyholders is quasi nonexistent and thus the surrender rate (independent individual decisions) can be modeled thanks to the Gaussian distribution whose mean and standard deviation are those observed. Indeed the suitable distribution in Region 1 is the classical Normal distribution represented in Figure 2.

On the contrary the sharp rise of the surrender rate at some level $\Delta r$ in Figure 1 , followed by a flat plateau which is the maximum reachable surrender rate (this bound is often suggested by an expert since we consider that we have never observed it), reflects that economical conditions are deteriorating. The crucial point is to realize that in such a situation the assumption of independent behaviors can become strongly erroneous: the correlation between policyholders' decisions makes the surrender rate distribution change. This is the consequence of two different behaviors or scenarii, either almost all policyholders surrender their contract or they don't. The more suitable distribution to explain it is the so-called Bi-modal distribution illustrated in Figure 2.

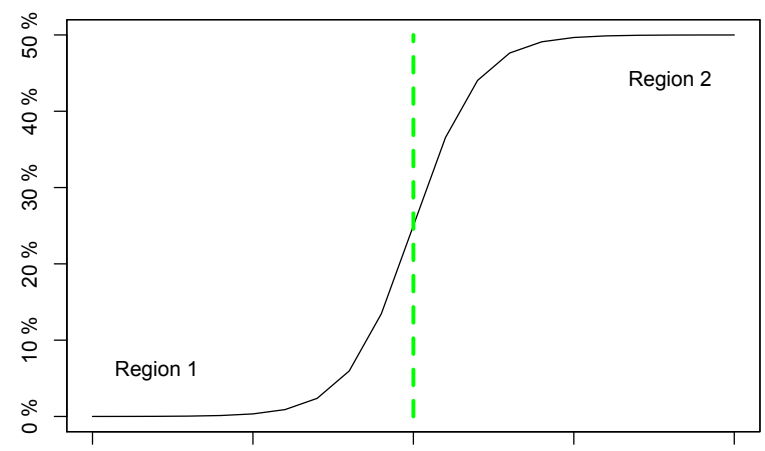

Figure 1. Surrender rate versus $\Delta \mathrm{r}$. 

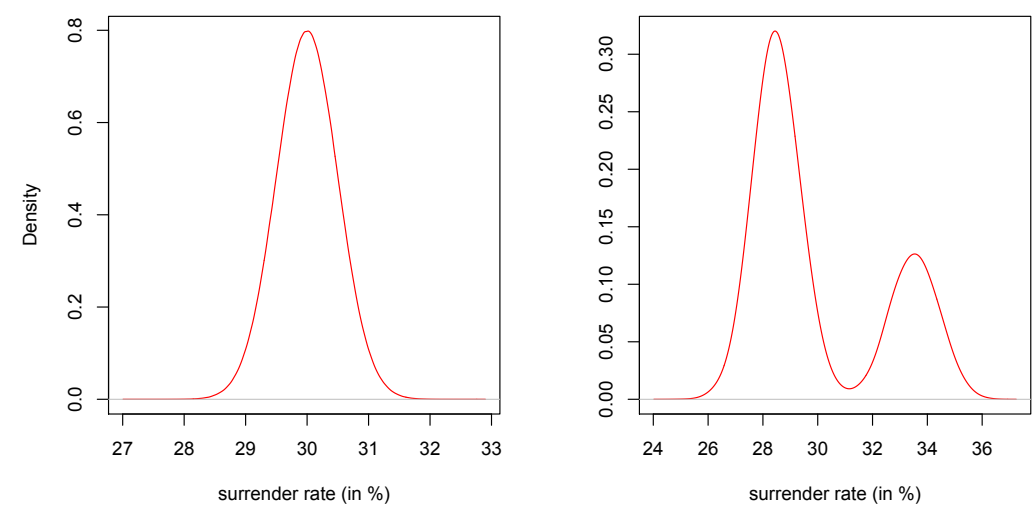

Figure 2. On the left, the density of a Gaussian distribution and on the right a Bi-modal density (mean equals 30).

The main difference of these two models (Gaussian and Bi-modal) is that the mean of the surrender rate is the result of two peaks of the density, what is extremely dangerous for the insurer who can thus experiment unexpected very high surrender rates causing big losses.

Note that irrational behavior of policyholders could also lead to correlation crises between their decisions even if $\Delta r$ is small. We shall see that this situation is the one that has the strongest impact on economic capital needs.

\section{Distribution of surrender rates}

\subsection{Combinatorial approach}

Consider a portfolio of $n \geq 2$ policyholders. Denote by

$$
N=\sum_{k=1}^{n} J_{k}
$$

be the number of copycat policyholders, and by

$$
M=\sum_{k=1}^{n} I_{k}
$$

the number of policyholders who surrender their contracts. Recall that

$$
I_{k}=J_{k} I_{0}+\left(1-J_{k}\right) I_{k}^{\perp},
$$

where $J_{k}$ corresponds to the indicator that the $k^{\text {th }}$ policyholder follows the market consensus (copycat behavior), where the random variable $J_{k}$ follows 
a Bernoulli distribution whose parameter $p_{0}$, and where $I_{0}, I_{1}^{\perp}, I_{2}^{\perp}, \ldots$ are independent, identically distributed random variables with parameter $p$. If copycat behavior is surrender (when $I_{0}=1$ ), then for $M$ to be equal to some integer $k \in[0, n]$, the number $N$ of copycat policyholders has to be smaller or equal to $k$, otherwise one would have $M \geq N>k$. Similarly, if copycat behavior corresponds to no surrender $\left(I_{0}=0\right)$, then for $M$ to be equal to some integer $k \in[0, n]$, the number $N$ of copycat policyholders has to be smaller or equal to $n-k$, otherwise one would have $M \leq n-N<n-(n-k)=k$. From the total probability formula, for $0 \leq k \leq n$, one has

$$
\begin{aligned}
P(M=k) & =P\left(M=k \mid I_{0}=0\right) P\left(I_{0}=0\right)+P\left(M=k \mid I_{0}=1\right) P\left(I_{0}=1\right) \\
& =\sum_{i=0}^{k} P\left(M=k \mid I_{0}=1, N=i\right) P\left(I_{0}=1, N=i\right) \\
& +\sum_{j=0}^{n-k} P\left(M=k \mid I_{0}=0, N=j\right) P\left(I_{0}=0, N=j\right) .
\end{aligned}
$$

From the mutual independence of the $\left(I_{k}\right)_{k \geq 0}$ and $\left(J_{l}^{\perp}\right)_{l \geq 1}$, for $0 \leq k \leq n$ we have

$$
P(M=k)=p \sum_{i=0}^{k} a_{i, k}+(1-p) \sum_{j=0}^{n-k} b_{j, k},
$$

where for $0 \leq i \leq k$,

$$
a_{i, k}=C_{n}^{i} p_{0}^{i}\left(1-p_{0}\right)^{n-i} C_{n-i}^{k-i} p^{k-i}(1-p)^{n-k},
$$

and for $0 \leq j \leq n-k$ we have

$$
b_{j, k}=C_{n}^{j} p_{0}^{j}\left(1-p_{0}\right)^{n-j} C_{n-j}^{k} p^{k}(1-p)^{n-j-k} .
$$

Note that for fixed $k$, the $a_{i, k}, 0 \leq i \leq k$ and the $b_{j, k}, 0 \leq j \leq n-k$ can be computed than to the following recursive formulas: for $0 \leq i \leq k$, we have

$$
\frac{a_{i+1, k}}{a_{i, k}}=\frac{C_{n}^{i+1}}{C_{n}^{i}} \frac{p_{0}}{p\left(1-p_{0}\right)} \frac{C_{n-i-1}^{k-i-1}}{C_{n-i}^{k-i}}=\frac{k-i}{i+1} \frac{p_{0}}{p\left(1-p_{0}\right)}
$$

and for $0 \leq j \leq n-k$, we have

$$
\frac{b_{j+1, k}}{b_{j, k}}=\frac{C_{n}^{j+1}}{C_{n}^{j}} \frac{p_{0}}{(1-p)\left(1-p_{0}\right)} \frac{C_{n-j-1}^{k}}{C_{n-j}^{k}}=\frac{n-j-k}{j+1} \frac{p_{0}}{(1-p)\left(1-p_{0}\right)} .
$$

Note that as

$$
a_{0}=b_{0}=\left(1-p_{0}\right)^{n} C_{n}^{k} p^{k}(1-p)^{n-k}
$$

is generally quite small, it is often preferable to start by $a_{i_{0}}$ and $b_{j_{0}}$ such that $a_{i_{0}}$ and $b_{j_{0}}$ are large enough in order to minimize round-off errors. Some efficient algorithms and associated accuracy to make this kind of computations can be found in Viquerat (2010). 
a )

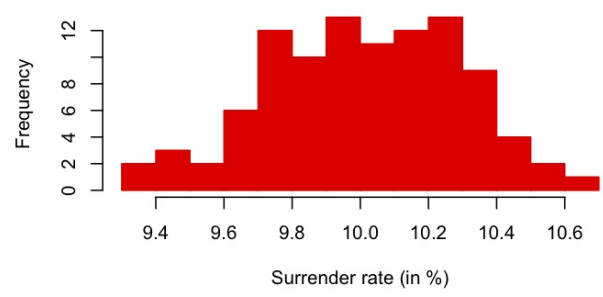

c)

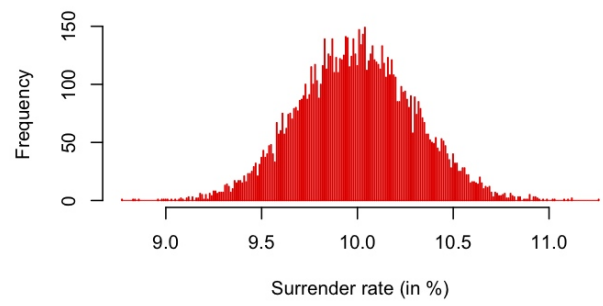

b )

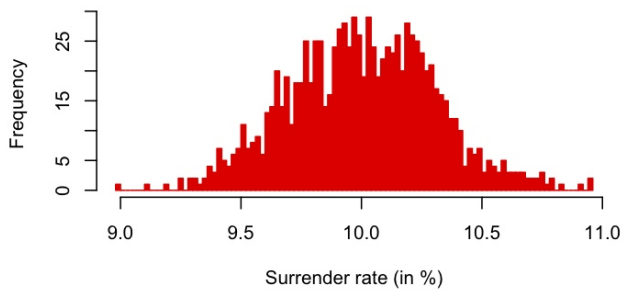

d)

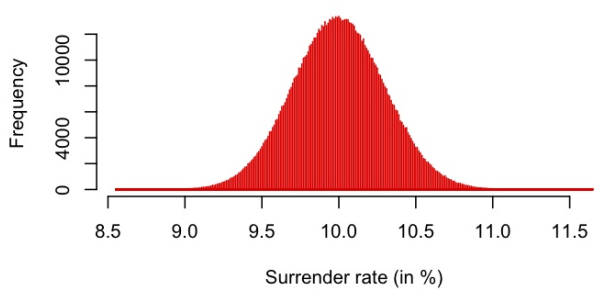

Figure 3. Effect of the number of simulations on the surrender rate distribution: a) 100, b) 1 000, c) 10000 and d) 1000 000. No copycat behavior, individual surrender probability equals $10 \%, 10000$ policyholders in the portfolio.

\subsection{Simulation approach}

Another possibility is to use Monte Carlo simulations, particularly if surrender risk is one of the numerous risks considered in a complex internal risk model. Below is a test of the effects of various parameters on the probability distribution of the surrender rate of some parameters like the number of simulations, the portfolio size, the mean probability to surrender and the correlation. The
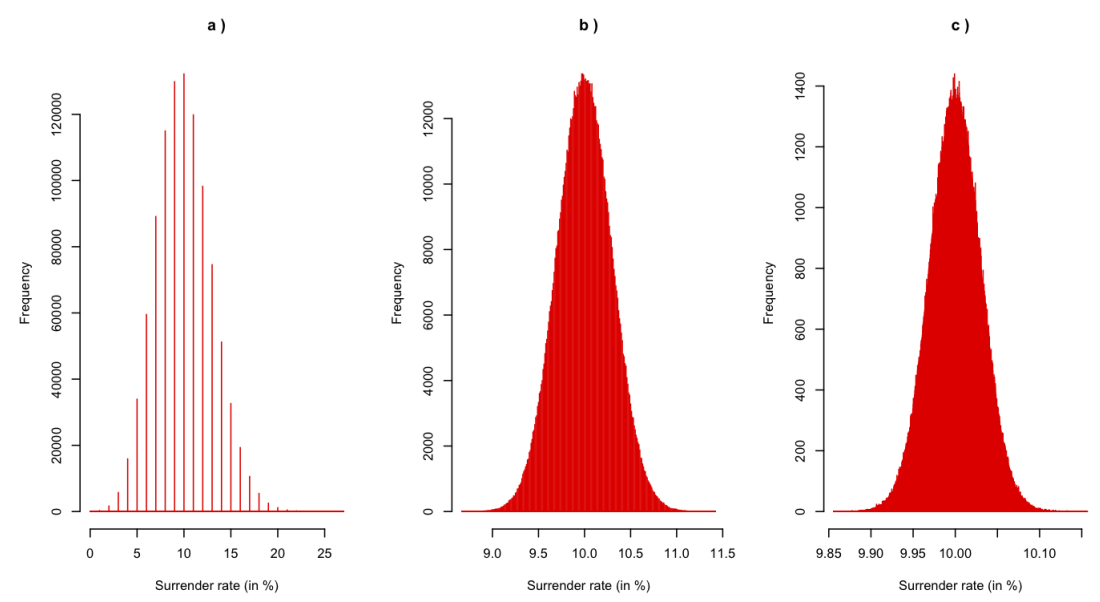

Figure 4. Effect of the portfolio size on the surrender rate distribution: a) 100 policyholders in the portfolio, b) 10000 and c) 1000 000. No copycat behavior, individual surrender probability equals 10\%, 1000000 simulations. 

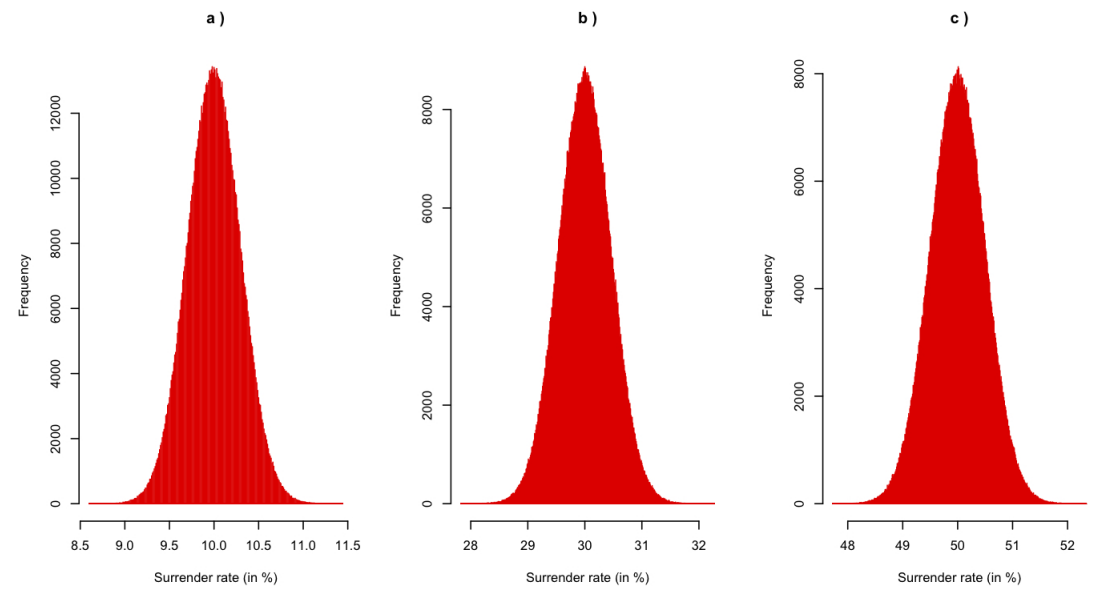

Figure 5. Rebalancing the surrender rate distribution: individual surrender probability equals: a) $10 \%$, b) $30 \%$, and c) $50 \%$. No correlation (copycat surrender probability is equal to 0), 10000 insureds and 1000000 simulations.

number of simulations is of course a key factor to get an accurate and consistent approximation of the surrender rate distribution whatever the socioeconomic context. The number of policyholders in the portfolio is somewhat important because the range of surrender rate values narrows (less dispersion), although it does not really affect the shape of the distribution. Figure 3 and Figure 4 corroborate these observations and that is why hereafter one sets the number of simulations to 1000000 and the number of policyholders to 10 000. Figure 5 shows that the effect of the individual surrender probability $(p)$

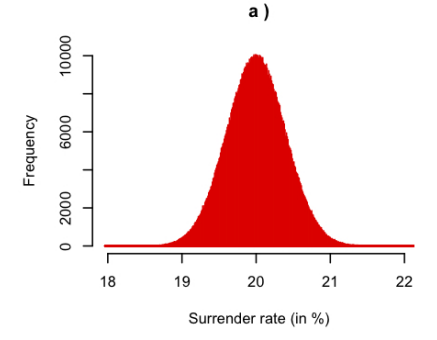

d)

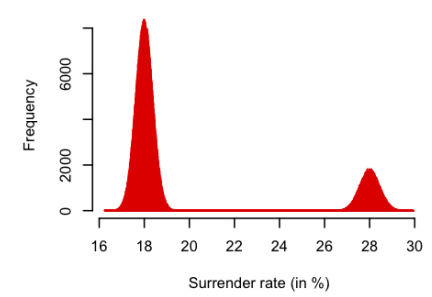

b)
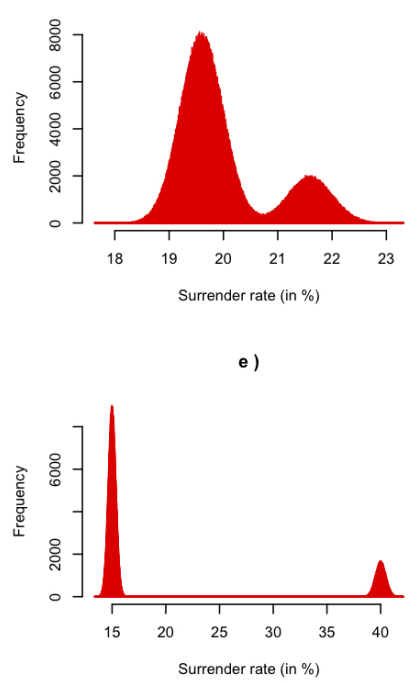

c)

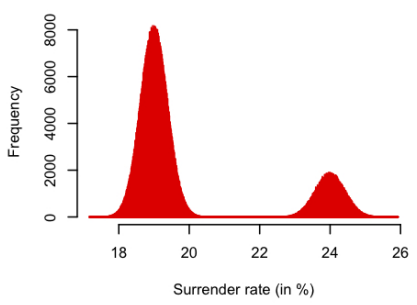

f)

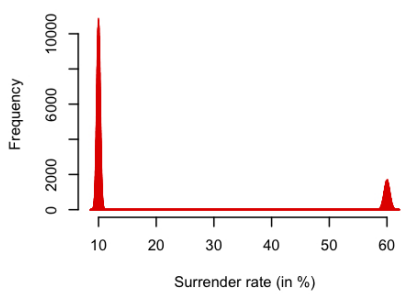

Figure 6. Reshaping the surrender rate distribution (Gaussian to Bi-modal). From top left to bottom right, copycat behavior probability equals: a) $0 \%$, b) $2 \%$, c) $5 \%$, d) $10 \%$, e) $25 \%$ and f) $50 \%$. Individual surrender probability equals $20 \%, 1000000$ simulations and 10000 policyholders. 


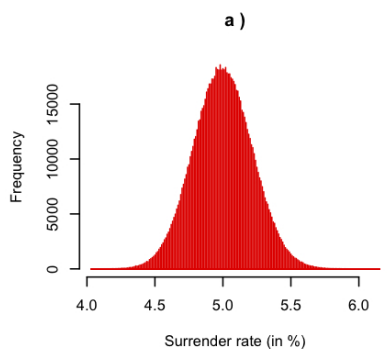

d)

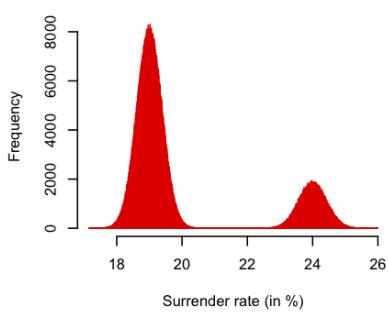

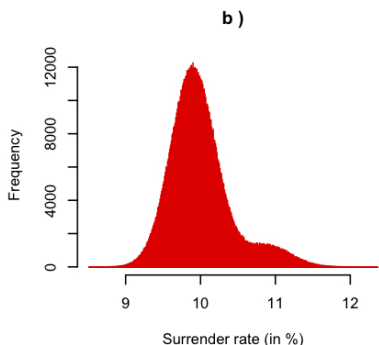

e)

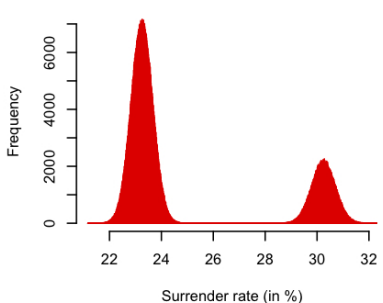

c)

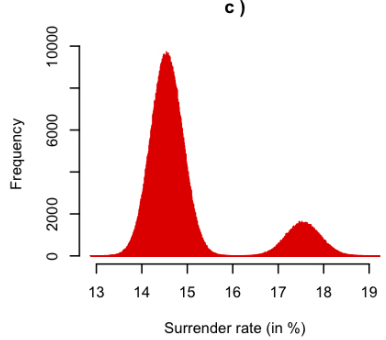

f)

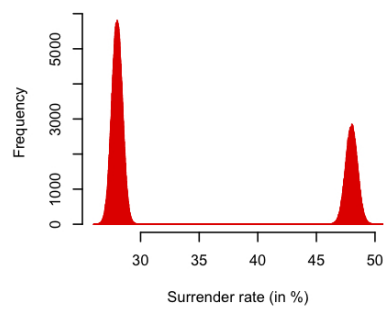

Figure 7. Distribution of the surrender rate depending on the economy. From top left to bottom right, both correlation and surrender probability increase: individual surrender probability and copycat behavior probability respectively equal to a) $5 \%$ and $0 \%$, b) $10 \%$ and $1 \%$, c) $15 \%$ and $3 \%$,d) $20 \%$ and $5 \%$, e) $25 \%$ and $7 \%$, f) $35 \%$ and $20 \%$.

is major but not surprising. It strongly impacts the mean of the distribution causing the density to be greater at high values, and so provoking the portfolio to have a risky profile.

Let us now focus on the consequences of varying the correlation, the core of this paper. The correlation parameter represented by the probability $p_{0}$ to make the same decision (surrender or not) plays the main role: Figure 6 shows that increasing the correlation considerably reshapes the distribution of the surrender rate.

Actually both the surrender probability and the correlation increase during an economic crisis, and this is no good news for the insurer who is faced to a much more frightening situation when looking at the distribution of the surrender rate in Figure 7. These graphs confirm the theory developed in the previous section.

We may notice that for a given $\Delta r$ (and thus for a given $p_{0}$ in theory), the bigger the correlation parameter $p_{0}$ is, the more the surrender density shape becomes bi-modal. The insurer is interested in quantifying the difference between these distributions in terms of behavioral risk. Risk measures will help us to get indicators of the gap between these distributions. More precisely, he would like to compute the well-known Value-at-Risk or VaR, defined for the random variable $\mathrm{X}$ and the threshold $\alpha$ as follows:

$$
\operatorname{VaR}_{\alpha}(X)=\inf \left\{x \in X, F_{X}(x) \geq \alpha\right\}
$$




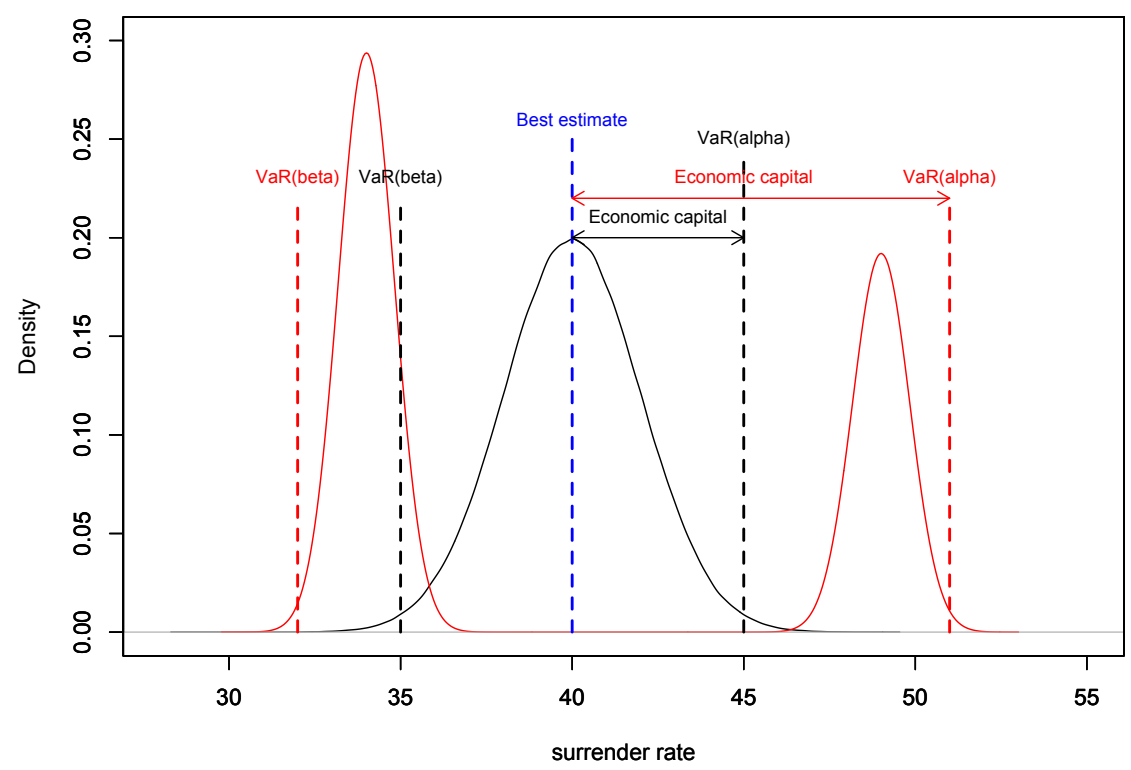

Figure 8. Risk measures and economic capital in Normal and Bi-modal models. Independent behaviors (black line) causes the economic capital reserves to be much lower than with correlated behaviors (red line).

In our application, the random variable $X$ represents the surrender rate. This means that the insurer expects to experiment a surrender rate lower than $\mathrm{VaR}$ with $\alpha \%$ confidence. In life insurance, the classical threshold is set to $\alpha=99.5 \%$.

Sometimes the insurer also fears that the surrender rate suddenly drops, because the size of its portfolio may become too large with respect to its capital constraints, and because interest rate levels might be unfavorable to the insurer. In this case, the configurations defined above have to be adapted, as illustrated on Figure 8 where the risk measures that are interesting for the insurer are $V a R_{\alpha}$ (right-hand side, risk of massive surrenders) and $V a R_{\beta}$ (lefthand side, risk that policyholders surrender their contract much more seldom than expected).

\section{Qualitative comparison with stochastic orders}

Denote by $M_{\left(p, p_{0}\right)}$ the number of policyholders who surrender their contract in the above model when $P\left(J_{1}=1\right)=p_{0}$ and when $P\left(I_{1}=1\right)=p$. Let us examine how the values of $p$ and $p_{0}$ affect the distribution of the conditional surrender rate. We use the concept of $s$-convex stochastic ordering (see Lefèvre \& Utev (1996) and Denuit et al. (1998)). Given two random variables $Y$ and 
$Z$, for any $s=1,2, \ldots$, we have

$X \leq_{s-c x}^{\mathcal{D}} Y$ if $E[\phi(Y)] \leq E[\phi(Z)]$ for all $s$-convex function $\phi: \mathcal{D} \rightarrow \mathbb{R},(1)$

i.e. in short, for any function $\phi$ on $\mathcal{D}$ whose $s$-th derivative exists and satisfies $\phi^{(s)} \geq 0$. Note that the first $s-1$ moments of $Y$ and $Z$ are then necessarily equal. The order $\leq_{1-c x}^{\mathcal{D}}$ is just the usual stochastic order $\leq_{1}, \leq_{2-c x}^{\mathcal{D}}$ is the usual convex order $\leq_{2}$ (which implies in particular $\operatorname{Var}(Y) \leq \operatorname{Var}(Z)$ ). In addition, $X$ is said to be smaller than $Y$ in the increasing convex order (denoted by $\left.\leq_{i c x}\right)$ if

$$
E(f(X)) \leq E(f(Y))
$$

for every increasing convex function such that the expectation is defined.

Proposition 1 When the correlation parameter is fixed, the number of surrenders is stochastically increasing in $p$ : for fixed $p_{0} \in(0,1)$, if $p<p^{\prime}$ then we have

$$
M_{\left(p, p_{0}\right)} \leq_{1} M_{\left(p^{\prime}, p_{0}\right)}
$$

Proof: The result follows from elementary, classical results about stochastic orderings of Bernoulli and Binomial distributions.

Proposition 2 When the individual surrender probability $p$ is fixed, the correlation parameter induces a 2-convex ordering of numbers of surrenders: for fixed $p \in(0,1)$, if $p_{0}<p_{0}^{\prime}$ then we have

$$
M_{\left(p, p_{0}\right)} \leq_{2} M_{\left(p, p_{0}^{\prime}\right)}
$$

Proof: Given that the number of copycat policyholders $N$ is equal to $k$, the total number of surrenders reads

$$
M_{(p, k)}=k \cdot I_{0}+0 . I_{1}^{\perp}+0 . I_{2}^{\perp}+\ldots+0 . I_{k}^{\perp}+1 . I_{k+1}^{\perp}+\ldots+1 . I_{n}^{\perp} .
$$

Given that $N=k^{\prime}$ with $k \leq k^{\prime}$, we have

$$
M_{\left(p, k^{\prime}\right)}=k^{\prime} \cdot I_{0}+0 . I_{1}^{\perp}+\ldots+0 . I_{k}^{\perp}+\ldots+0 . I_{k^{\prime}}^{\perp}+1 . I_{k^{\prime}+1}^{\perp}+\ldots+1 \cdot I_{n}^{\perp} .
$$

The two random variables $M_{(p, k)}$ and $M_{\left(p, k^{\prime}\right)}$ may be compared with respect to the majorization order (see e.g. Marshall \& Olkin (1979)). For any vector $Z$, denote by $Z^{\downarrow}=\left(z_{1}^{\downarrow}, \ldots, z_{K}^{\downarrow}\right)$ the vector with the same components, but sorted in decreasing order. Given two vectors $Y=\left(y_{1}, \ldots, y_{K}\right)$ and $Z=\left(z_{1}, \ldots, z_{K}\right)$ of size $K \geq 1$ such that

$$
\sum_{i=1}^{K} y_{i}=\sum_{i=1}^{K} z_{i}
$$


recall that $Z$ is said to majorize $Y$ if for all $j \leq K$,

$$
\sum_{i=1}^{j} y_{i}^{\downarrow}=\sum_{i=1}^{j} z_{i}^{\downarrow} .
$$

From Marshall \& Olkin (1979), if the vector $\alpha=\left(\alpha_{0}, \ldots, \alpha_{n}\right)$ is lower than the vector $\beta=\left(\beta_{0}, \ldots, \beta_{n}\right)$ in the Majorization partial order, and if the $X_{i}$ are independent and identically distributed, then we get the following convex ordering:

$$
\sum_{i} \alpha_{i} X_{i} \leq_{2} \sum_{i} \beta_{i} X_{i}
$$

Here we set $X_{i}=I_{i}^{\perp}$ and

$$
\left(\alpha_{0}, \ldots, \alpha_{n}\right)=(k, \underbrace{0, \ldots, 0}_{k \text { times }}, 1, \ldots, 1) \text { and }\left(\beta_{0}, \ldots, \beta_{n}\right)=(k^{\prime}, \underbrace{0, \ldots, 0}_{k^{\prime} \text { times }}, 1, \ldots, 1) .
$$

For $k \leq k^{\prime}$, the vector $\left(\beta_{0}, \ldots, \beta_{n}\right)$ clearly majorizes the vector $\left(\alpha_{0}, \ldots, \alpha_{n}\right)$. Moreover, the random variable $\left(N \sim \operatorname{Bin}\left(n, p_{0}\right)\right)$ is stochastically increasing in $p_{0}$. So we can conclude that for $p_{0} \leq p_{0}^{\prime}$,

$$
M_{\left(p, p_{0}\right)} \leq_{2} M_{\left(p, p_{0}^{\prime}\right)}
$$

where $M_{\left(p, p_{0}\right)}$ denotes the number of policyholders who surrenders when the copycat probability is $p_{0}$ and when the individual surrender probability is $p$.

Proposition 3 In the model where both $p$ and $p_{0}$ increase with $\Delta r, \Delta r$ induces an increasing-convex ordering of numbers of surrenders: denote by $M$ (resp. $M^{\prime}$ ) the number of surrenders when $\Delta r=x$ (resp. $\left.\Delta r=x^{\prime}\right)$. If $x<x^{\prime}$ then we have

$$
M \leq_{i c x} M^{\prime}
$$

Proof: We use the same arguments as in the proof of Propositions 1 and 2. A combination of these arguments yields to this proposition because when $\Delta r$ increases, both $p$ and $p_{0}$ increase.

Proposition 1 implies that the expected value, the Value-at-Risk of any level $\alpha \in(0,1)$ and the stop-loss premiums $E\left[(M-m)_{+}\right]$for $0 \leq m \leq n$ are increasing in $p$ and in $\Delta r$. Proposition 2 implies that the variance and the stop-loss premiums $E\left[(M-m)_{+}\right]$for $0 \leq m \leq n$ are increasing in $p_{0}$ (when $p$ is fixed). It also shows that if $p_{0}<p_{0}^{\prime}$, there exists some level $\alpha_{0} \in(0,1)$ such that for $\alpha>\alpha_{0}$,

$$
\operatorname{VaR}_{\alpha}\left(M_{\left(p, p_{0}\right)}\right)<\operatorname{Va} R_{\alpha}\left(M_{\left(p, p_{0}^{\prime}\right)}\right)
$$

because the Karlin-Novikov cut criterion says in that case that the cumulative distribution functions of $M_{\left(p, p_{0}\right)}$ and $M_{\left(p, p_{0}^{\prime}\right)}$ cross only one time. 


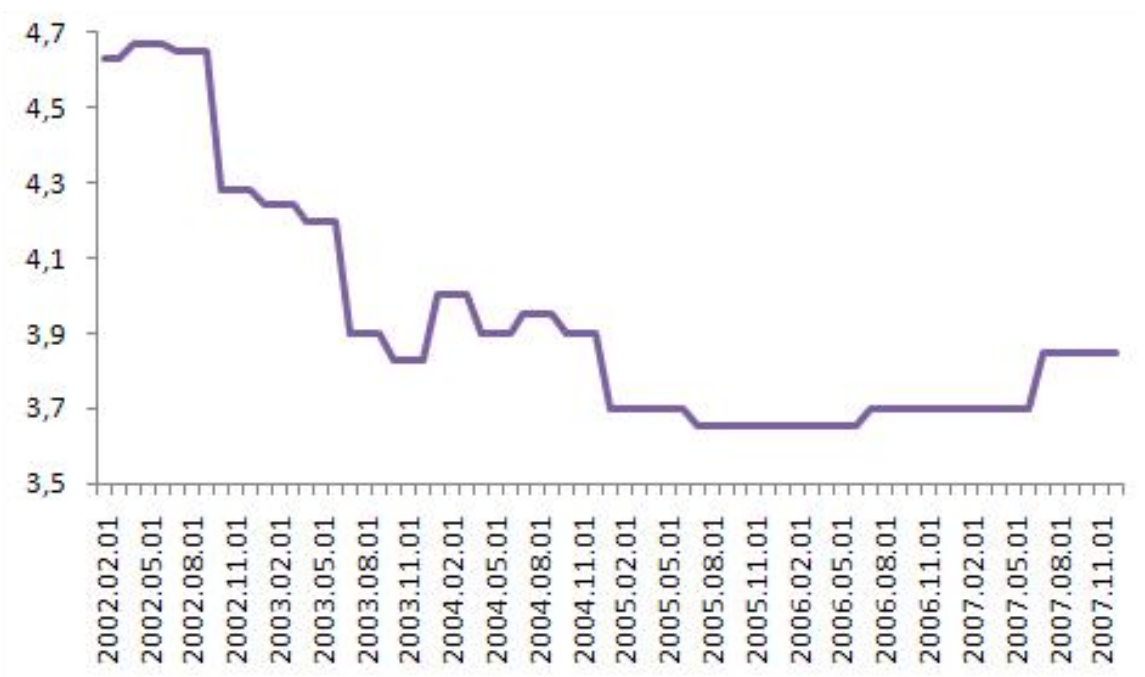

Figure 9. Evolution of the difference between the credited rate and the risk-free interest rate on the market (universal).

It is not surprising that, in this model, increasing the correlation parameter and/or the marginal surrender probability leads to a higher measure of risk in general. But the purpose of this paper is also to determine the importance of the impact of correlation on economic capital requirements and on the so-called account value introduced in the next section, for a real-world life insurance portfolio.

\section{Application on a real life insurance portfolio}

This section does not aim at giving accurate results that are valid for all insurance portfolios, but it illustrates how to implement in practical terms all the theory developed previously. To quantify exactly the impact of correlated behaviors on final economic capital of the company requires a good calibration of all the parameters used in the following, and thus a rather complete and reliable database. This is why all statements and computations of this section have to be moderated.

Products in the scope of this study are savings products extracted from the Spanish reinsurance portfolio of a global life reinsurer.

Monthly data cover the period ranging from February 2002 to December 2007. The number of policyholders in the portfolio varies from 291 at the beginning of the observation period to 25766 in July 2006. On average, there are 17657 policyholders in the portfolio each month. The professionals of the insurance sector usually consider that, to be relevant, $\Delta r$ should stand for the credited rate of the competitors on the same business line minus the one of the contract 


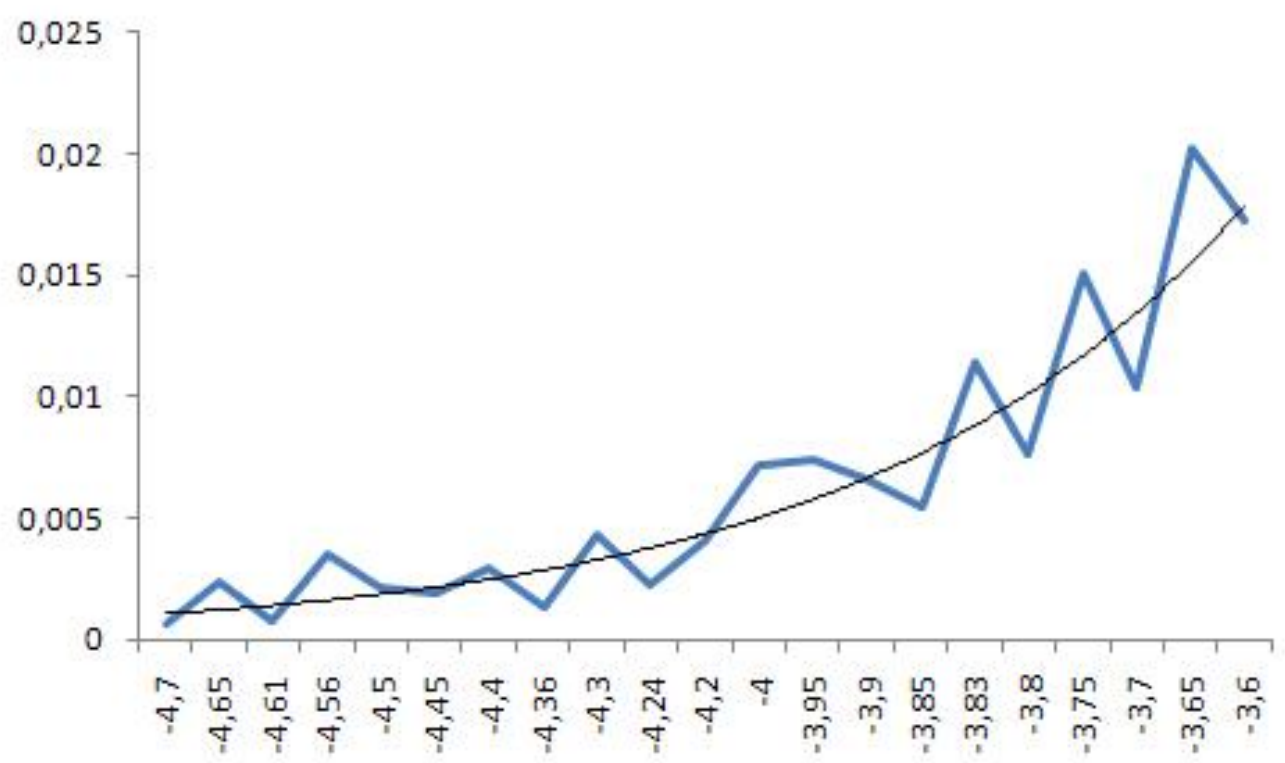

Figure 10. Monthly surrender rate versus $\Delta r$, the difference between the risk-free rate and the credited rate.

(Milhaud, Loisel \& Gonon (2010)). This information are difficult to obtain. To overcome this problem, some experts agree to say that the credited rate of the competitors somewhat equals to the risk-free rate plus a constant $c$, so that

$$
\Delta r=(\text { risk-free rate }+c)-\text { credited rate. }
$$

In our application, we set $c=0$ and this is the way we define $\Delta r$. As you can see on Figure 9, the credited rate of the contract is greater than the risk-free rate during all the period, which yields to $\Delta r<0$. An exponential smoothing of the surrender rate in function of $\Delta r$ on this (proprietary) data leads Figure 10: the smaller this difference, the higher the surrender rate. This is quite normal since it means that when the credited rate decreases, $\Delta r$ tends to 0 and the contract becomes less advantageous. The exponential smoothing has no importance except that it is useful to recognize the S-shaped curve.

As discussed in the first section, one only observes market conditions that correspond to Region 1 in Figure 1. Here we assume that the maximum reachable monthly surrender rate (flat plateau in Region 2 of Figure 1) corresponding to the "expert opinion" is equal to $3.5 \%$ a month. Notice that this is another source of error if the expert is wrong. Of course, the calibration is arbitrary here. To build a better model to forecast surrender rates in another story. Here, we are mainly interested in the impact of correlation between policyholders and we take this model, used by some insurance companies, as a realistic illustrative example.

Denote by $Y$ the random variable that represents the surrender rate. The empirical mean of the surrender rate in Region 1, denoted by $\tilde{Y}_{n_{1}}$, equals 
$\tilde{Y}_{n_{1}}=\frac{1}{n_{1}} \sum_{i=1}^{n_{1}} Y_{i, 1}$, where $n_{1}$ is the number of observations $Y_{i, 1}$ in Region 1.

The empirical standard deviation, denoted by $\tilde{\sigma}_{n_{1}}$, is given as usual by the following unbiased estimator:

$$
\tilde{\sigma}_{n_{1}}=\sqrt{\frac{1}{\left(n_{1}-1\right)} \sum_{i=1}^{n_{1}}\left(Y_{i, 1}-\tilde{Y}_{n_{1}}\right)^{2}} .
$$

Here the numerical application gives $\tilde{Y}_{n_{1}}=6.73 \times 10^{-3}$ and $\tilde{\sigma}_{n_{1}}=4.28 \times 10^{-3}$. The variability coefficient, denoted by $\tilde{\nu}_{n_{1}}$, is defined by $\tilde{\nu}_{n_{1}}=\frac{\tilde{\sigma}_{n_{1}}}{\tilde{Y}_{n_{1}}}$.

In Region 1, $\tilde{\nu}_{n_{1}}$ equals $63.5 \%$, which reflects that data are quite highly dispersed. Anyway, these statistics are clearly not tolerable as they are based on a very low number of observations but this dispersion also appears because of cohort effects conjugated to tax constraints. The effect of the date with time dependence can also play a role here.

Because we do not have data for very high levels of the surrender rate, we assume that the variability coefficient remains constant whatever the surrender rate average. This assumption enables us to compute an approximation of the standard deviation in Region 2, which is $\tilde{\sigma}_{n_{2}}=\frac{\tilde{Y}_{n_{2}}}{\tilde{Y}_{n_{1}}} \tilde{\sigma}_{n_{1}} \simeq 0.022$. This choice of value is clearly arbitrary, but it would be difficult to come up with a more

a )

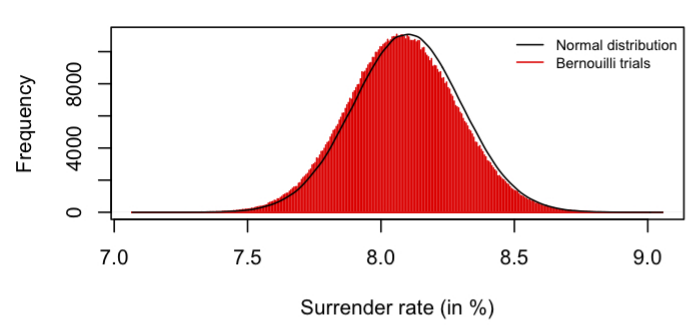

c)

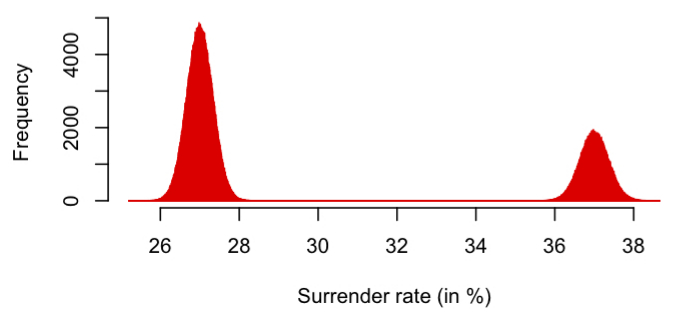

b)

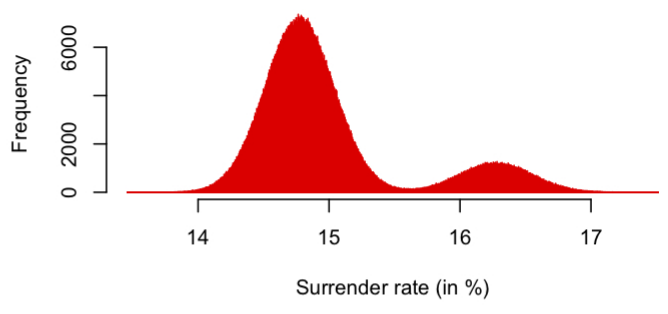

d)

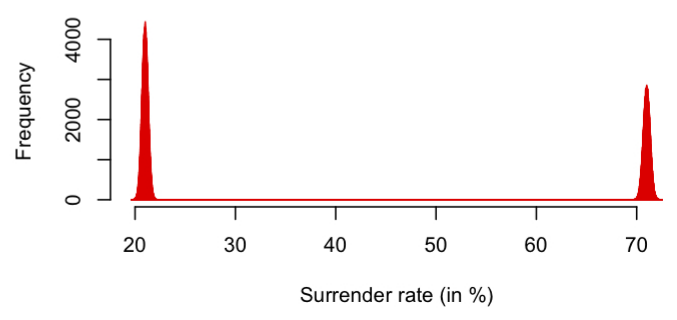

Figure 11. Evolution of surrender rate distribution depending on the economic context (1 000000 simulations, 17657 policyholders). Individual surrender probabilities and copycat behavior probabilities: a) $8.08 \%$ and $0 \%$ (theoretical Normal distribution in black), b) $15 \%$ and $1.5 \%$, c) $30 \%$ and $10 \%$ and d) $42 \%$ and $50 \%$. 
objective choice.

Thus we simulate in Region 1 Bernoulli trials with parameter $p_{1}$ equal to $\tilde{Y}_{n_{1}}$ and check that the distribution of the surrender rate is close to a Normal distribution with mean $\tilde{Y}_{n_{1}}$ and standard deviation $\tilde{\sigma}_{n_{1}}$. Finally, the $k^{\text {th }}$ policyholder makes the decision $I_{k} \sim \operatorname{Bernoulli}\left(p_{1}\right)$, and the Bernoulli indicator $J_{k}$ that she follows the market consensus $J_{k}$ has parameter $p_{0}=0$ (independent decisions because we are in Region 1).

In Region 2, $I_{k} \sim \operatorname{Bernoulli}\left(p_{2}\right)$ (with $p_{2}=\tilde{Y}_{n_{2}}=0.035$ ) and the existing correlation impacts the parameter $p_{0}$ of $J_{k}$. Indeed one sets $J_{k} \sim \operatorname{Bernoulli}\left(p_{0}\right)$, with $p_{0}=0.5$. The number of simulations in all this section is set to 1000000 and the number of policyholders is set to 17657 . Figure 11 shows the results of these simulations: on the top left, the simulation of behaviors confirms the Gaussian approximation when considering independent Bernoulli trials. The Normal distribution $\mathcal{N}\left(m=\tilde{Y}_{n_{1}}, \sigma=\tilde{\sigma}_{n_{1}}\right)$ fits well and the calibration of the standard deviation does not look so bad. On the bottom right, it is clear that the Gaussian approximation is not at all appropriated to model policyholders' behavior in that case! Other graphs highlight the transition between these two caricatural situations.

Remark: we have converted monthly surrender rates to yearly ones because $V a R$ is usually estimated for a 1-year period in insurance. Time dependence could also be considered and may have a strong impact on the overall surrender risk faced by the company. Nevertheless we focus here on a 1-period framework and keep the multi-period extension for further research. This yields to $\tilde{Y}_{n_{1}} \simeq$ $0.0808=8.08 \%, \tilde{\sigma}_{n_{1}} \simeq 0.05, \tilde{Y}_{n_{2}} \simeq 0.42=42 \%$ and $\tilde{\sigma}_{n_{2}} \simeq 0.264$.

The impact of the economical context on $V a R$ is quite impressive: for instance if the correlation increases from $0 \%$ to $50 \%$ in a "soft" context, the $V a R_{99.5 \%}$ increases by $510 \%$ (difference denoted by $\Delta V a R_{99.5 \%}$ ). It means that if the insurer wants to be covered against a very high surrender rate and takes correlation crises into account, the cost of capital might be about $510 \%$ higher than expected!

Table 1 summarizes it for different economical contexts, levels of correlation and levels of $V a R$.

Detailed analysis of the $V a R$ deviations: Let us focus on the $99.5 \%$ $\operatorname{VaR}$ because this is the $\operatorname{VaR}$ of interest in Solvency II. Figure 12 shows how $V a R$ is affected by an increase in correlation between policyholders' decisions. For a given individual probability to surrender, $1 \%$ say, a shift in correlation from 0 to $1 \%$ increases $\operatorname{VaR}_{99.5 \%}$ by 30 to $50 \%$.

Notice that large positive deviations are mainly concentrated for small variations in correlation when considering low surrender propensity. This result is quite interesting and we decided to define classes in terms of sensitivity (with 


\begin{tabular}{|c|c|c|c|c|c|}
\hline Economic context & Correlation value & $V a R_{90 \%}$ & $V a R_{95 \%}$ & $V a R_{99.5 \%}$ & $\Delta V a R_{99.5 \%}$ \\
\hline \multirow{7}{*}{ soft $(p=8.08 \%)$} & $p_{0}=0$ & 8.704763 & 8.784052 & 9.055899 & $0 \%$ \\
\hline & $p_{0}=0.01$ & 9.441015 & 9.571275 & 9.735516 & $+7.5 \%$ \\
\hline & $p_{0}=0.02$ & 10.33584 & 10.48309 & 10.72662 & $+18.5 \%$ \\
\hline & $p_{0}=0.05$ & 13.15059 & 13.31483 & 13.54137 & $+49.5 \%$ \\
\hline & $p_{0}=0.15$ & 22.46135 & 22.63125 & 22.87478 & $+152.6 \%$ \\
\hline & $p_{0}=0.3$ & 36.34819 & 36.52376 & 36.80693 & $+306.4 \%$ \\
\hline & $p_{0}=0.5$ & 54.81679 & 55.00368 & 55.28119 & $+510.4 \%$ \\
\hline \multirow{7}{*}{ Medium $(p=20 \%)$} & $p_{0}=0$ & 20.89823 & 21.01150 & 21.27768 & $0 \%$ \\
\hline & $p_{0}=0.01$ & 21.50988 & 21.66846 & 21.93464 & $+3.1 \%$ \\
\hline & $p_{0}=0.02$ & 22.22914 & 22.44436 & 22.72187 & $+6.8 \%$ \\
\hline & $p_{0}=0.05$ & 24.64745 & 24.86266 & 25.13451 & $+18.1 \%$ \\
\hline & $p_{0}=0.15$ & 32.70658 & 32.92745 & 33.20496 & $+56.1 \%$ \\
\hline & $p_{0}=0.3$ & 44.78111 & 45.00198 & 45.27383 & $+112.8 \%$ \\
\hline & $p_{0}=0.5$ & 60.74645 & 60.95599 & 61.24483 & $+187.8 \%$ \\
\hline \multirow{7}{*}{$\operatorname{Hard}(p=42 \%)$} & $p_{0}=0$ & 43.11604 & 43.25763 & 43.46152 & $0 \%$ \\
\hline & $p_{0}=0.01$ & 43.49550 & 43.67673 & 43.93725 & $+1.1 \%$ \\
\hline & $p_{0}=0.02$ & 43.98822 & 44.22042 & 44.52059 & $+2.4 \%$ \\
\hline & $p_{0}=0.05$ & 45.69859 & 45.95911 & 46.32724 & $+6.6 \%$ \\
\hline & $p_{0}=0.15$ & 51.51498 & 51.77550 & 52.16062 & $+20 \%$ \\
\hline & $p_{0}=0.3$ & 60.16877 & 60.41796 & 60.75777 & $+39.8 \%$ \\
\hline & $p_{0}=0.5$ & 71.74492 & 71.97712 & 72.28295 & $+66.3 \%$ \\
\hline
\end{tabular}

Table 1

Estimations of Value-at-Risk for the surrender rate with different correlations.

respect to correlation):

- very sensitive configuration (red area on Figure 12 ): $p \in] 0,0.05]$ and $p_{0} \in$ ]0, 0.1];

- sensitive configuration (orange area): $p \in] 0,0.05]$ and $p_{0} \in[0.1,0.4]$, or $p \in] 0.05,0.2]$ and $\left.\left.p_{0} \in\right] 0,0.3\right]$;

- low sensitivity configuration (yellow area): other situations.

In the very sensitive configuration, the $V a R_{99.5 \%}$ can increase by up to $70 \%$ ! In the sensitive configuration, the insurer may face less adverse movements but is faced to a potential increase by 5 to $25 \%$ of $V a R_{99.5 \%}$. Finally, the low sensitivity configuration allows the insurer to be confident because the $V a R$ that she has chosen is often high enough. 


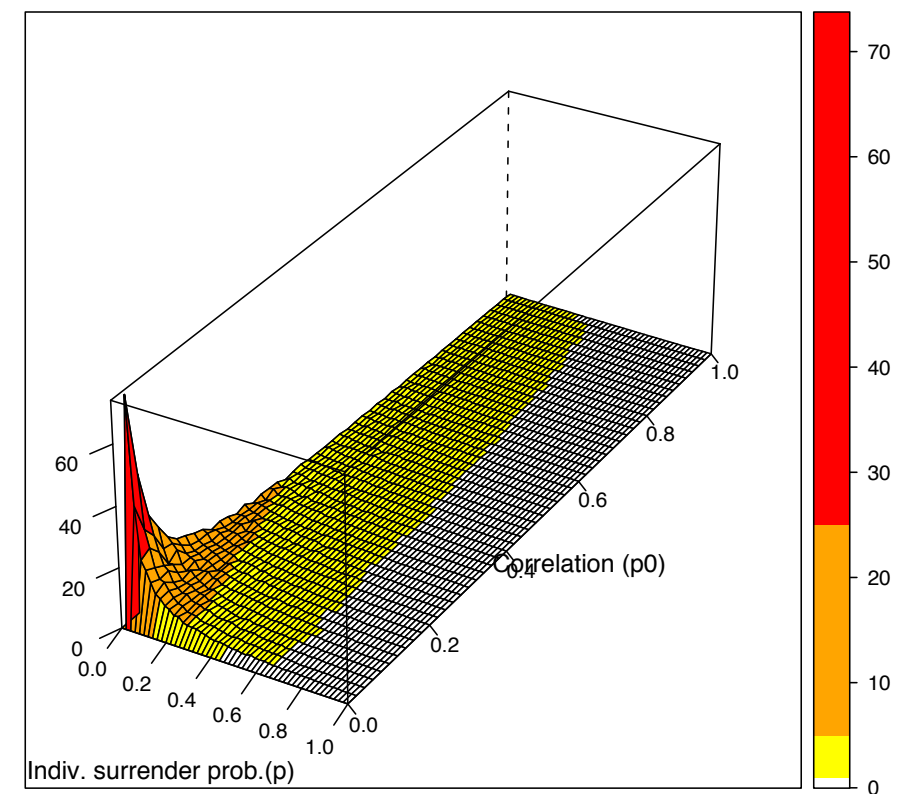

Figure 12. Relative deviation (in \%) of the $V a R$ versus $p_{0}$ and $p$.

\section{Gap between standard assumptions and a more realistic model?}

How do the surrender assumptions affect the funds reserves ? We focus in this section on the consequences on insurer reserves when considering erroneous surrender rate distribution, where policyholders' behaviors are considered to be quite homogeneous (around a "mean" behavior) and independent, instead of introducing correlation. In some insurance groups, each entity may define how to integrate the surrender assumptions and modeling into the pricing of the product, and the estimations of its future profitability. Sometimes several definitions are quite different even within a single entity, depending on the product. To fix ideas, we shall assume that the so-called Account Value (AV) is adjusted with withdrawals so that the funds returns are re-balanced. A quite classical way for insurers to manage surrenders is to set base surrenders and then to adjust it dynamically. The point is that this adjustment is still based on independent behaviors of policyholders, which is precisely what we suggest to avoid in this paper. Consider the simplified but realistic model of adjusted $\mathrm{AV}$ :

$$
A V_{a d j}(t)=A V_{a d j}(t-1) \times[1+\% \text { periodical benefit } \times(1-\text { surrender rate })]
$$

It is now possible to estimate the sensibility of the Account Value in terms of behavioral risk by using Monte Carlo simulations of various scenarii, corresponding to surrender shocks. This is precisely the recommendations of Solvency II which stimulates the development of internal risk models and the use of multiple scenarii to assess robust risk margins, the so-called economic capital (EC), which is the difference between the $V a R$ and the best estimate 


\begin{tabular}{|c|c|c|c|c|c|c|}
\hline Scenario & $\mathrm{BE}$ & $\mathrm{EC} / \mathrm{AV}\left(V_{a} R_{99.5 \%}^{\text {Normal }}\right)$ & Correlation & $\mathrm{EC}\left(\operatorname{VaR}_{99.5 \%}^{\text {Bi-modal }}\right)$ & $\Delta E C$ & $\Delta A V$ \\
\hline \multirow{4}{*}{$\begin{array}{c}\text { Soft } \\
(p=8.08 \%)\end{array}$} & \multirow{4}{*}{$\begin{array}{c}8.06 \% \\
\left(p_{0}=0\right)\end{array}$} & \multirow{4}{*}{$\begin{array}{c}0.84 \% / 1091029 \\
\left(p_{0}=0\right)\end{array}$} & $p_{0}=0.05$ & $5.45 \%$ & $4.61 \%$ & 5506.7 \\
\hline & & & $p_{0}=0.15$ & $14.85 \%$ & $14.01 \%$ & 14857.1 \\
\hline & & & $p_{0}=0.3$ & $28.7 \%$ & $27.86 \%$ & 28743.9 \\
\hline & & & $p_{0}=0.5$ & $47.26 \%$ & $46.42 \%$ & 47189.9 \\
\hline \multirow{4}{*}{$\begin{array}{l}\text { Medium } \\
(p=20 \%)\end{array}$} & \multirow{4}{*}{$\begin{array}{l}20.03 \% \\
\left(p_{0}=0\right)\end{array}$} & \multirow{4}{*}{$\begin{array}{c}1.18 \% / 1078824 \\
\left(p_{0}=0\right)\end{array}$} & $p_{0}=0.05$ & $5.06 \%$ & $3.88 \%$ & 5179.8 \\
\hline & & & $p_{0}=0.15$ & $13.15 \%$ & $11.97 \%$ & 13182.3 \\
\hline & & & $p_{0}=0.3$ & $25.35 \%$ & $24.17 \%$ & 25290.8 \\
\hline & & & $p_{0}=0.5$ & $41.3 \%$ & $40.12 \%$ & 41233.5 \\
\hline \multirow{4}{*}{$\begin{array}{c}\text { Hard } \\
(p=42 \%)\end{array}$} & \multirow{4}{*}{$\begin{array}{l}42.02 \% \\
\left(p_{0}=0\right)\end{array}$} & \multirow{4}{*}{$\begin{array}{c}1.48 \% / 1056459 \\
\left(p_{0}=0\right)\end{array}$} & $p_{0}=0.05$ & $4.28 \%$ & $2.8 \%$ & 4253.6 \\
\hline & & & $p_{0}=0.15$ & $10.13 \%$ & $8.65 \%$ & 10036 \\
\hline & & & $p_{0}=0.3$ & $18.76 \%$ & $17.28 \%$ & 18769.1 \\
\hline & & & $p_{0}=0.5$ & $30.21 \%$ & $28.73 \%$ & 30260.3 \\
\hline
\end{tabular}

Table 2

Estimations of differences of economic capital (in terms of surrender rate) and account value with different correlations in various scenarii.

(BE) (the $\mathrm{BE}$ is our expectation). The bi-modal distribution of surrender rate implies significant changes in these estimations and so in reserving: first the account value is estimated with best estimate assumptions and then with the $V a R_{99.5 \%}$ in the case of Normal distribution (independent behaviors) and Bi-modal distribution (correlated behaviors). The difference of estimated economic capitals represents the gap of reserves for the insurer in terms of lapse rate.

Numerical application The model used to introduce dependence between policyholders' behaviors is described in first section. Hereafter, assume that:

- the risk term is one year ;

- the economical and financial conditions are getting worse ;

- today, the account value equals 1000000 US\$;

- the yearly benefit is $10 \%$;

- the $V a R, E C$ and $A V$ are computed for the coming year.

Those parameters were chosen to reflect reality without betraying confidentiality. The portfolio size is still 17657 and the number of simulations is set to 1000 000. Table 2 highlights the potential impact of surrender modeling on the estimations of reserves on the consider products. It summarizes the differences of reserves in $\$$ for different economical contexts and levels of correlation. The results tell us that in a soft context, considering independent behaviors instead of $50 \%$ correlated behaviors can cause the insurer to experiment an unexpected loss up to $47190 \$$ of the account value at the end of the year ( $4.72 \%$ of the initial wealth)! The absolute difference between the mean 


\begin{tabular}{|c|c|c|c|c|c|c|}
\hline Portfolio size & $\mathrm{BE}$ & $\mathrm{EC} / \mathrm{AV}\left(V a R_{99.5 \%}^{\text {Normal }}\right)$ & Correlation & EC $\left(\right.$ VaR $\left.R_{99.5 \%}^{\text {Bi-modal }}\right)$ & $\Delta E C$ & $\Delta A V$ \\
\hline Little: & \multirow{3}{*}{$\begin{array}{c}8.1 \% \\
\left(p_{0}=0\right)\end{array}$} & \multirow{3}{*}{$\begin{array}{c}1.76 \% / 1090140 \\
\left(p_{0}=0\right)\end{array}$} & $p_{0}=0.05$ & $6.26 \%$ & $4.5 \%$ & 6280 \\
\hline 5000 & & & $p_{0}=0.2$ & $20.42 \%$ & $18.66 \%$ & 20440 \\
\hline policyholders & & & $p_{0}=0.5$ & $48.54 \%$ & $46.78 \%$ & 48560 \\
\hline Medium: & \multirow{3}{*}{$\begin{array}{c}8.08 \% \\
\left(p_{0}=0\right) \\
\end{array}$} & \multirow{3}{*}{$\begin{array}{c}0.51 \% / 1091408 \\
\left(p_{0}=0\right)\end{array}$} & $p_{0}=0.05$ & $5.1 \%$ & $4.59 \%$ & 5102 \\
\hline 50000 & & & $p_{0}=0.2$ & $19.01 \%$ & $18.5 \%$ & 19012 \\
\hline policyholders & & & $p_{0}=0.5$ & $46.63 \%$ & $46.12 \%$ & 46634 \\
\hline Big: & \multirow{3}{*}{$\begin{array}{c}8.08 \% \\
\left(p_{0}=0\right)\end{array}$} & \multirow{3}{*}{$\begin{array}{c}0.1426 \% / 1091777 \\
\left(p_{0}=0\right)\end{array}$} & $p_{0}=0.05$ & $4.73 \%$ & $4.59 \%$ & 4731.2 \\
\hline 500000 & & & $p_{0}=0.2$ & $18.56 \%$ & $18.41 \%$ & 18565 \\
\hline policyholders & & & $p_{0}=0.5$ & $46.16 \%$ & $46.01 \%$ & 46158.6 \\
\hline
\end{tabular}

Table 3

Impact of portfolio size on account value in a soft context (100 000 simulations).

expected surrender rate and the stressed one is $47.26 \%$ !

Besides the initial account value has been set to $1000000 \$$, which is very low as compared to usual equity capitals of insurance companies. Guess what would be the actual loss of the insurer in such a casual situation...

The size of the company is also a key-factor: let us extract the part of Table 2 concerning the "soft" context and study the impact of the number of policyholders in the portfolio. Initially, there were 17657 policyholders. Some huge insurance companies may think that their size prevents them from experimenting such scenario because of mutualization. The analysis of Table 3 demonstrates that the number of policyholders does not have a strong influence on the computation of the risk margin. To reserve enough money to cover the correlation risk of surrender behaviors is very important in predicting capital needs: underestimating this risk could make shareholders dissatisfied because of unexpected important margin calls. Because undiversifiable risks are present, increasing the size of the portfolio would not reduce the impact of a correlation crisis on the considered quantities.

\section{Conclusion}

We have shown in this article that the impact of the surrender rate distribution is major on both predicting the economic capital needs and the reserves of the company. This distribution results from a different point of view on behaviors' modeling: from conditionally independent behaviors, we move to correlated policyholders' decisions that better reflect the reality in our opinion. For further research, it would be essential to explore the path-dependent aspect or memory properties of the surrender rate trajectory (the present value depends on the previous ones), which should be meaningful to forecast it and understand its dynamics. Of course it requires to have empirical data, which 
is not always the case in practice.

To look into the question of the feedback impact of massive surrenders on interest rates and inflation rates could also be interesting as it could create some kind of vicious circle. Another source of risk for the insurer are options embedded in the contracts that enable to change the underlying investment type: for example the policyholder can move his money from Unit-Link to Euro products (Loisel et al. (2010)) lately.

To sum up, the lapse risk is extremely complicated to study because it depends on many factors: policyholders' characteristics, personal desires and needs, contract features and time elapsed, economical and financial context (liquidity issues), social and cultural aspects, but also regulator decisions. A potential intervention of the regulator might require the company to suspend surrender payments until things calm down a bit in very adverse scenarios. Finally the insurer cannot control all this but her target is to settle the best Asset and Liabilities Management that he can for a robust and sustainable solvability ; and this can start by trying to "master" the behavioral risk !

\section{Acknowledgment}

The authors thank Claude Lefèvre and Areski Cousin for useful suggestions. This work is partially funded by the reinsurance company AXA Global Life and the ANR (reference of the French ANR project : ANR-08-BLAN-0314-01).

\section{References}

Biard, R., Lefèvre, C. \& Loisel, S. (2008), 'Impact of correlation crises in risk theory: Asymptotics of finite-time ruin probabilities for heavy-tailed claim amounts when some independence and stationarity assumptions are relaxed', Insurance: Mathematics and Economics 43(3), 412 - 421.

Denuit, M., Lefèvre, C. \& Shaked, M. (1998), 'The $s$-convex orders among real random variables, with applications', Math. Inequal. Appl. 1(4), 585-613.

Lefèvre, C. \& Utev, S. (1996), 'Comparing sums of exchangeable Bernoulli random variables', J. Appl. Probab. 33(2), 285-310.

Loisel, S. (2008), 'From liquidity crisis to correlation crisis, and the need for quanls in erm', pp. 75-77. in Risk Management: The Current Financial Crisis, Lessons Learned and Future Implications, Edited by the SOA, CAS and CIA.

Loisel, S., Arnal, P. \& Durand, R. (2010), Correlation crises in insurance and finance, and the need for dynamic risk maps in orsa. working paper.

Marshall, A. \& Olkin, I. (1979), Inequalities: Theory of Majorization and Its Applications, Academic Press, New York. 
McNeil, A., Frey, R. \& Embrechts, P. (2005), Quantitative Risk Management, Princeton Series In Finance.

Milhaud, X., Loisel, S. \& Gonon, M.-P. (2010), D'une courbe de taux de rachat déterministe à une approche stochastique dans un modèle interne : prise en compte des crises de corrélation. working paper.

Milhaud, X., Loisel, S. \& Maume-Deschamps, V. (2010), Surrender triggers in life insurance : classification and risk predictions. working paper.

Viquerat, S. (2010), On the efficiency of recursive evaluations in relation to risk theory applications, $\mathrm{PhD}$ thesis. 